\title{
Numerical modeling of the salt-transfer problem in soils
}

\author{
Mazhid Yusupov ${ }^{1 *}$, Raikhan Tazhibayeva ${ }^{3}$, Sholpan Ziyaeva ${ }^{2}$, and Kurash Kubyashev ${ }^{2}$ \\ ${ }^{1}$ Chirchik State Pedagogical University, Tashkent, Uzbekistan \\ ${ }^{2}$ Tashkent institute of irrigation and agricultural mechanization engineers, Tashkent, Uzbekistan \\ ${ }^{3}$ International Kazakh-Turkish University named after H.A. Yasawi, Higher school of public \\ administration and Economics, Turkestan, Kazakhstan
}

\begin{abstract}
The article discusses the urgent problem associated with solving the problems of salt transfer in soils and is devoted to numerical modelling of the transference and diffusion of salts in soils, in which the total porosity of the soil is divided by the fraction of "through" and "deadend" pores with the corresponding concentration of the solution. A brief review of scientific publications devoted to this problem is given. To study and predict the process of the spread of harmful substances, a mathematical model and a numerical algorithm for computer experimentation have been developed. The solution was obtained using a method based on the use of quadrature formulas in combination with the differential sweep method for a system of ordinary second-order differential equations with arbitrary linear boundary conditions. The concentrations of salts in the "through" and "dead-end" pores, respectively, were studied at various values of the filtration process. The results are shown in the graphs.
\end{abstract}

\section{Introduction}

The analysis of the studies of the process of moisture and salt transfer in soil showed that when irrigated with water with a salinity of up to $15 \mathrm{~g} / 1$, crop yields can be obtained for several years. The relative well-being of the reclamation state of lands irrigated with mineralized waters is temporary for 2-3 years. Their long-term use in many cases leads to serious consequences, manifested not so much in the form of the toxic effect of salts, but in the form of sharp changes in the physicochemical properties of soils, increased alkalinity, and deterioration of water-physical properties of soils as a result of shale formation and fusion.

Based on the foregoing, research and management decisions on moisture and salt transfer in soils is an urgent problem to maintain the state of soils for growing agricultural products.

One of the effective tools for predicting and monitoring the state of the soil is a mathematical tool: a model, a numerical algorithm, and software tools that can be used to run a computer experiment on a computer when setting the basic hydrochemical and

*Corresponding author: kubyashev1983@mail.ru 
hydrodynamic parameters of the soil. To solve the above problem, a study was conducted, and significant results of applied and fundamental nature were treated.

In particular, in [1] the problem associated with the urgent task of the process of moisture and salt transfer in the soil is solved, and a review of scientific papers devoted to various aspects and mathematical support of the object of study is given. To carry out a comprehensive study, a mathematical model is proposed with allowance for the pore ground cohesion with fine particles over time; changes in the coefficient of soil permeability, water loss and filtration coefficient; changes in the initial porosity and porosity of the settled mass, as well as an effective numerical algorithm based on the Samarsky-Fryazinov vector scheme with the second order of approximation of differential operators to finite-difference. To derive a mathematical model of salt transfer, it is proposed that the pressure gradient in the channel is constant and equal to atmospheric pressure.

In [2], hydrodynamic and hydraulic models of water runoff in wetlands were proposed, allowing one to describe the processes of filtration and surface runoff with varying degrees of detail and accuracy. Based on the models of salt transfer by interacting filtration and channel flows, modelling the quality of groundwater and surface water is considered.

In [3], a problem is considered related to filtration and dehydration of liquid and ionic solutions from gel particles and heavy ionic compounds. This process is implemented to prepare and purify chemical solutions, drinking water, pharmaceuticals, liquid fuels, general products, etc. A mathematical model has been developed to analyze, research, determine the main parameters of the technological process and the operating modes of filter units, and support managerial decision-making. Using the developed model, a series of computational experiments on a computer was carried out.

In [4], a problem is considered related to dehydration and filtering of liquid ionic solutions from gel particles and heavy ionic compounds. The specified technological process is implemented during the preparation and purification of chemical solutions, drinking water, pharmaceuticals, liquid fuels, common products, etc. To analyze, research, determine the main parameters of the technological process, as well as the operating modes of the filter units and, ultimately, support management decisions, a mathematical model was developed in which various operating modes of the filter units and the physicochemical properties of ionic solutions can be taken into account. Since the developed mathematical model of an unsteady technological process of filtering complex suspensions is described by a system of nonlinear partial differential equations, it is difficult to obtain its analytical solution.

In [5], for the numerical integration of the problem to suppress small-scale oscillations, a term of artificial viscosity was added, and the nonlinear terms of the equation are linearized by the quasilinearization method of Bellman-Kalaba.

In [6], a mathematical model was developed for predicting the groundwater level in two-layer formations. For mathematical modelling of the geofiltration process, a two-layer medium consisting of two layers is considered: soil (with low throughput) and water.

The salt solution in the soil is in two physical states: in the form of a solution firmly bound by molecular forces to the surfaces of the particles and as a free solution occupying the space inside the pore cavities. It was shown in [7] that the assessment of the washing process as a process of displacing in the soil of the salt solution with washing water is valid for structureless, dusty and non-saline soils. Based on soil washing experiments, it was found that salt removal from pre-moistened soil is reduced. This is because during capillary hydration, the salt efflorescences gradually dissolve and are absorbed into the unit together with moisture.

The movement of salts and moisture in the soil is a complex physical and chemical process. Along with the displacement of a free solution by diffusion transfer of salts, the dissolution of salts of the solid phase, and an exchange of salts occurs between the free pore 
solution and intra-unit moisture. The three-parameter model distinguishes between pore solutions located in "dead ends" and "through" pores. In the total porosity $\mathrm{m}$ of the soil, we distinguish the fraction of "through" pores $\mathrm{k}$ with the concentration of solution $\mathrm{C}$ and the fraction of "dead-end" pores (1-k) with the concentration of solution N. The "through" pores, which are active in the filtration ratio, exchange salts with "dead ends". We denote the rate of macro transport in "through" pores by $\theta$, and the convective diffusion coefficient referred to the surface unit of "through" pores, D.

\subsection{The problem formulation. Consider the following mathematical model of the problem of salt transfer in soil on reclaimed lands [8-9]:}

$$
\left\{\begin{array}{c}
k \frac{\partial C}{\partial t}+\theta k \frac{\partial C}{\partial x}+(1-k) \alpha(C-N)=D k \frac{\partial^{2} C}{\partial x^{2}} \\
\frac{\partial N}{\partial t}=\beta(C-N)
\end{array}\right.
$$

where $\mathrm{C}$ and $\mathrm{N}-(\mathrm{g} / \mathrm{l}) ; \beta$ is parameter characterizing the intensity of the exchange between components $\mathrm{C}$ and $\mathrm{N}$ (1/day); $\alpha$ is a parameter characterizing the exchange of salts of active and stagnant zones and additionally takes into account sorption effects in "through" pores (at $\alpha \neq \beta)(1 /$ day).

Various solutions of system (1) are possible, for example, under the following initial and boundary conditions:

$$
\begin{aligned}
& \left\{\begin{array}{lll}
C=\tilde{C}_{0} & \text { at } & t=0 \\
N=\widetilde{N}_{0} & \text { at } & t=0
\end{array}\right. \\
& \left\{\begin{array}{lll}
C=C_{n} & \text { at } & x=0 \\
\frac{d C}{d x}=0 & \text { at } & x=L
\end{array}\right.
\end{aligned}
$$

$\mathrm{Cn}$ is the concentration of wash water.

\section{Materials and Methods}

The method for solving the problem (1) - (3) is based on the use of quadrature formulas in combination with the differential sweep method.

Introducing in (1) - (3) the following dimensionless quantities

$$
\bar{x}=\frac{x}{L}, \quad \bar{t}=\frac{D}{L^{2}} t, \quad \bar{C}=\frac{C}{C_{x}}, \quad \bar{N}=\frac{N}{C_{x}}, \quad \tilde{C}_{01}=\frac{\tilde{C}_{0}}{C_{x}}, \quad \overline{C_{n}}=\frac{C_{n}}{C_{x}}, \quad \overline{N_{01}}=\frac{\widetilde{N}_{0}}{C_{x}}
$$

while maintaining the same notation and accepting

$$
\mu_{1}=\frac{\theta L}{D}, \quad \mu_{2}=\frac{L^{2} \alpha}{D k}, \quad \mu_{3}=\frac{L^{2} \beta}{D}
$$

we get

$$
\left\{\begin{array}{c}
\frac{\partial C}{\partial t}+\mu_{1} \frac{\partial C}{\partial x}+\mu_{2}(1-k)(C-N)=\frac{\partial^{2} C}{\partial x^{2}} \\
\frac{\partial N}{\partial t}=\mu_{3}(C-N)
\end{array}\right.
$$


Assuming $\mathrm{t}_{\mathrm{m}}=(\mathrm{m}-1) \Delta \mathrm{t},(\mathrm{m}=1,2.3 \ldots)$ we integrate the system (4) with respect to $t$ in the interval $\left[0, t_{\mathrm{m}}\right]$ and using the quadrature formulas of trapezium, we have

$$
\left\{\begin{array}{c}
C_{m}-\tilde{C}_{0}+\mu_{1} \sum_{i=1}^{m} A_{i} \frac{d C_{i}}{d x}+\mu_{2}(1-k) \sum_{i=1}^{m} A_{i}\left(C_{i}-N_{i}\right)=\sum_{i=1}^{m} A_{i} \frac{d^{2} C_{i}}{d x^{2}} \\
N_{m}-\widetilde{N}_{0}=\mu_{3} \sum_{i=1}^{m} A_{i}\left(C_{i}-N_{i}\right)
\end{array}\right.
$$

where $\Delta t$ is the time step, $\mathrm{A}_{\mathrm{i}}$ is the entire quadrature formula of the trapezium.

After some transformations in (5), we can obtain the following system of equations

$$
\left\{\begin{array}{c}
\frac{d^{2} C_{m}}{d x^{2}}+a \cdot \frac{d C_{m}}{d x}+b \cdot C_{m}(x)=f_{m}(x) \\
N_{m}(x)=k_{6 m} \cdot A_{m} \cdot\left(\sum_{i=1}^{m-1} A_{i} C_{i}-\sum_{i=1}^{m-1} A_{i} N_{i}\right)+k_{7 m}+k_{6 m} \cdot C_{m}(x)
\end{array}\right.
$$

where

$$
\begin{gathered}
a=-\mu_{1}, \quad b=-\mu_{2}\left(1-k+\frac{1}{\mu_{2} A_{m}}+\frac{\mu_{2}(1-k) \mu_{3} A_{m}}{\left(1+\mu_{3} A_{m}\right)}\right) \\
k_{1 m}=-\frac{1}{A_{m}}, \quad k_{2 m}=\frac{\mu_{1}}{A_{m}}, \quad k_{3 m}=\frac{\mu_{2}(1-k)}{A_{m}}-\frac{\mu_{2}(1-k) \mu_{3}}{\left(1+\mu_{3} A_{m}\right)}, \quad k_{4 m}=-k_{3 m} \\
k_{5 m}=-\frac{\widetilde{N}_{0} \mu_{2}(1-k)}{\left(1+\mu_{3} A_{m}\right)}-\frac{1}{A_{m}} \tilde{C}_{0}, \quad k_{6 m}=\frac{\mu_{3} d t}{2\left(1+\mu_{3} A_{m}\right)}, \quad k_{7 m}=\frac{\widetilde{N}_{0}}{\left(1+\mu_{3} A_{m}\right)} . \\
f_{m}(x)=k_{1 m} \cdot \sum_{i=1}^{m-1} A_{i} \frac{d^{2} C_{i}}{d x^{2}}+k_{2 m} \cdot \sum_{i=1}^{m-1} A_{i} \frac{d C_{i}}{d x}+k_{3 m} \cdot \sum_{i=1}^{m-1} A_{i} C_{i}+k_{4 m} \cdot \sum_{i=1}^{m-1} A_{i} N_{i}+k_{5 m}
\end{gathered}
$$

First, the first equation of system (6) with boundary conditions

$$
\left\{\begin{array}{lll}
C_{m}=C_{n} & \text { at } & x=0 \\
\frac{d C_{m}}{d x}=0 & \text { at } & x=1
\end{array}\right.
$$

is solved by the differential sweep method [10,11], according to which the solution is sought in the form:

$$
\alpha_{m}^{\prime}(x) C_{m}^{\prime}(x)+\beta_{m}(x) C_{m}(x)=\gamma_{m}(x)
$$

where $\alpha_{m}(x), \beta_{m}(x), \gamma_{m}(x)$ - are the running coefficients, which are found from the solutions of the following Cauchy problem:

$$
\begin{aligned}
& \left\{\begin{array}{c}
\alpha_{m}^{\prime}(x)-a \cdot \alpha_{m}(x)+\beta_{m}(x)=0 \\
\beta_{m}^{\prime}(x)-\alpha_{m}(x) \cdot \beta_{m}(x)=0 \\
\gamma_{m}^{\prime}(x)=\alpha_{m}(x) \cdot f_{m}(x)
\end{array}\right. \\
& \left\{\begin{array}{c}
\alpha_{m}(0)=0 \\
\beta_{m}(0)=1 \\
\gamma_{m}(0)=C_{n}
\end{array}\right.
\end{aligned}
$$

Solving Cauchy problems (7), (8), we find: 


$$
C_{m}(1)=\gamma_{m}(1) / \beta_{m}(1) \text { and } C_{m}^{\prime}(1)=0
$$

Next, solving the equation

$$
\frac{d^{2} C_{m}}{d x^{2}}+a \cdot \frac{d C_{m}}{d x}+b \cdot C_{m}=f_{m}(x)
$$

under initial conditions (9), we find the salt concentration in the "through" pores $C_{m}(x)$ and substituting it in the second relation of the system (6), we determine the salt concentration in the "dead-end" pores $\mathrm{N}_{\mathrm{m}}(\mathrm{x})$.

The corresponding Cauchy problems can be solved by the fourth-order Runge-Kutta method [12].

\section{Results and Discussion}

Based on the developed algorithm, a computer program was compiled in which the obtained results are reflected in the form of graphs. During the calculation, the following initial data were used: $D=0.03 ; c_{0}=0.3 ; n_{0}=0.1 ; c_{n}=0.3 ; \theta=0.03 ; \alpha=0.4 ; \beta=0.06 ; k$ $=0.7$.

Figure 1 shows the influence of the coefficient $\mathrm{k}$ on the concentration of the solution of "through" pores $C(t)$ and on the concentration of the solution of "dead-end" pores $N(t)$. In the initial processes, the concentration of the solution of "through" pores decreases to its minimum and then slowly increases. The concentration of the solution of "dead end" pores $N(t)$ increases over time. With a decrease in $k$, the concentration of the solution also decreases.

Figure 2 shows the influence of the parameter $\alpha$ characterizing the exchange of salts of active and stagnant zones and also considers the sorption effects in "through" pores on the solution concentration. In this case, high-quality images are preserved by the change in salt concentration, but it occurs "insignificant" quantitative changes.
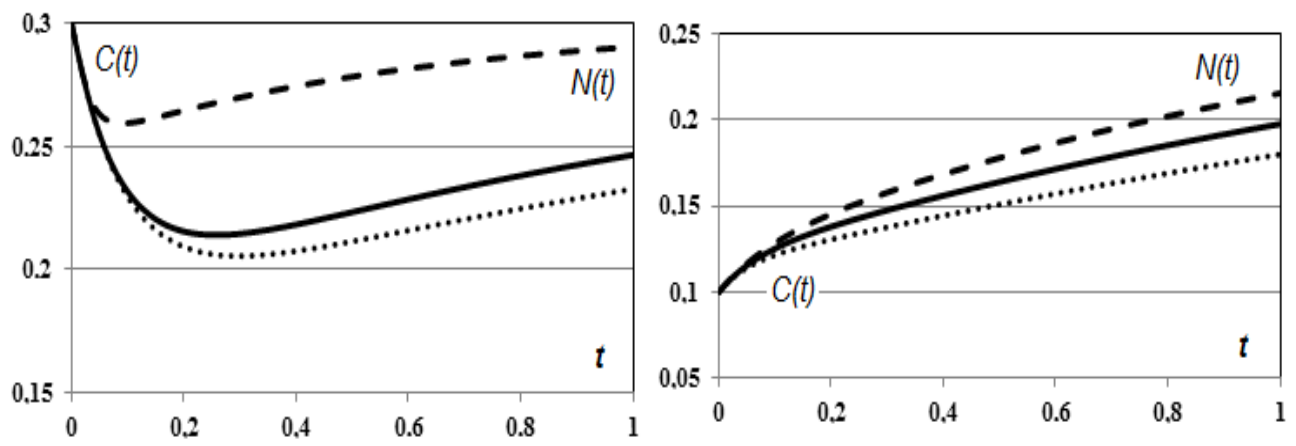

Fig. 1. $k=0.7$ (dashed), $k=0.6$ (solid), $k=0.5$ (dot) 

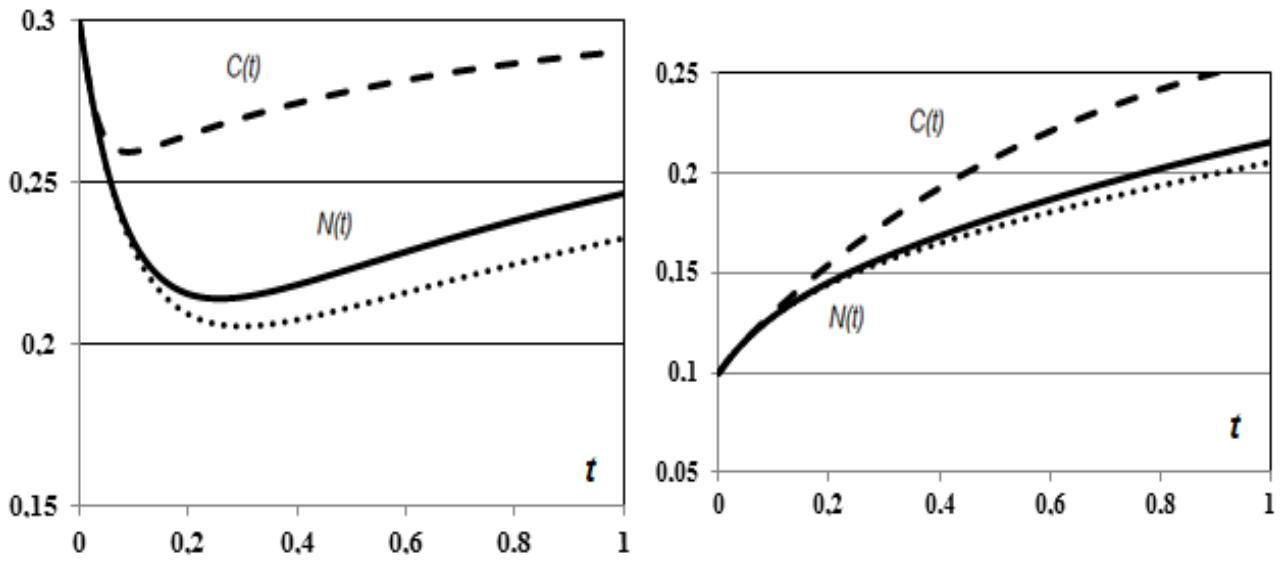

Fig. 2. $\alpha=0.2$ (dashed), $\alpha=0.4$ (solid), $\alpha=0.6$ (dot)

For various values of time $(\mathrm{t})$, the change in the salt concentration rate in the "through" pores over the entire pore space is shown (Figure 3). At the beginning of the pore space, the salt concentration rate increases with time, and at the end of the pore space, it will be zero.

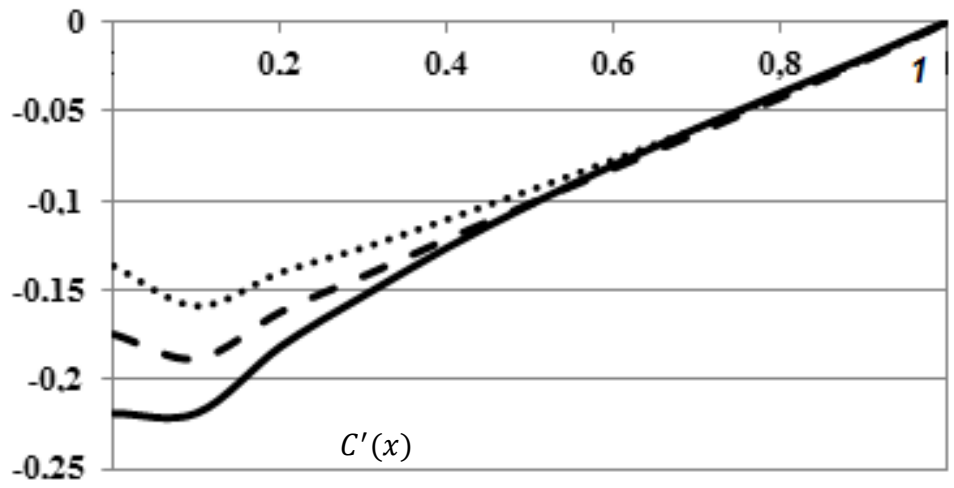

Fig. 3. The rate of salt concentration in the "through" pores of the pore space, $t=0.3$ (solid), $\mathrm{t}=0.5$ (dashed), $\mathrm{t}=0.7$ (dot).

\section{Conclusions}

Based on data analysis, it could be concluded that for woody and shrubby vegetation, the main features of the assessment of soil protection capacity are: the closeness (density) of tree crowns and shrub belt and the state of the forest floor and topsoil cover. Vegetation is of meadow-steppe type, with many herbs and shrubs, in places - juniper, spruce, apple, individual walnut trees. A practically intact vegetative community with good soil protection ability is observed in this area. The highest percentages of projective cover are characteristic of high turf. High- and medium-resistant species are found in the grassland. Communities of this category do not need special measures for restoration (except in insignificantly insolated eroded slopes). Rational use is all they require. From what has been said above, it follows that soils distributed on shadow slopes, with the exception of eroded differences, always have a powerful fine-grained cover, which does not contribute to the formation of surface runoff. Therefore, these sites are the best lands in mountainous areas. 
There are plant communities with insignificant signs of disturbance, such as reducing the percentage of turf cover (with a sufficiently high projective coverage) and the sites with weakly erosion-resistant species. Weak manifestation of erosion processes is observed (with the exception of eroded insolated slopes). These lands require partial surface improvement with the regulation of use. Vegetation of this category requires partial reforestation measures aimed at increasing the closeness of crowns and the restoration of wood floor and favorable conditions for the natural renewal of wood vegetation. Naturally, all plant communities need protection, continuous reforestation measures and facilitation in natural renewal.

\section{References}

1. Ravshanov N., Khurramov I., Aminov S.M., Mathematical modeling of the process of water-soline transport in soils, Journal of Phys.: Conf. Series 1210 (012118), (2019).

2. Kashevarov A.A., Modeling of water-drain and salt-transfer processes on swamped lands, Applieds Mechanics and Tech, (2005)

3. Ravshanov N., Saidov U.M., Modeling technological process of ion-exchange filtration of fluids in porous media, Conf. Ser. 1015 (032114), (2018)

4. Ravshanov N., Saidov U., A model for studying the non-stationary technological process of ion-exchange filtering of complex suspensions, Problems of computational and applied mathematics. T., pp. 33-41, (2016)

5. Ravshanov N., Saidov U., Model problem of the technological process of ionexchange filtering of a suspension and their numerical-analytical solution, Problems of computational and applied mathematics, Tashkent, (2018).

6. Ravshanov N., Daliev Sh., Mathematical model for predicting the groundwater level in two-layer strata, Information technology modeling and management.Voronezh, Scientific book, (2019)

7. Verigin N.N., Some issues of chemical hydrodynamics of interest for land reclamation and hydraulic engineering, Academy of Sciences of the USSR, OTN, Moskow, (1953)

8. Averyanov S.F., The fight against salinization of irrigated lands, Moskow.: Kolos, (1978).

9. Rex L.M., System studies of reclamation processes and systems, (Moskov.: Aslan, (1995).

10. Yusupov M., On an approach to solving nonlinear boundary value problems for second-order differential equations, Collection of abstracts of the conference "Integral Equations - 2009", Kiev, (2009).

11. M. Yusupov, B.O. Rahkmankulova and Sh.A.Aynakulov, Numerical solutions of the problem of salt-transfer in soils, E3S Web of Conferences, (2019)

12. Bakhvalov N.S., Numerical methods, Moskow.: Nauka, (1975)

13. Djamalov K., Rakhmankulova B., Noraliyev N.X., Ziyaeva Sh., Mathematical model of salt balance Stress-deformed state of ore-totropic spherical shell weakened by two circular holes, MPCPE-2020, (2020)

14. Yusupov M., Rakhmankulova B., Ziyaeva B., Kushaev A., Vehicle oscillation taking into account the rheological properties of the suspension, MPCPE-2020, (2020)

15. Rakhmankulova B., Nigmatov A.M., Akbaraliyev A.A., Improving system efficiency in automatic regulation of the water level in the drainage system, SCIENCE, RESEARCH, DEVELOPMENT, 31, pp. 23-25. (2020) 